\title{
A literature review on inventory modeling with reliability consideration
}

\author{
Imtiaz Ahmed* and Ineen Sultana
}

Department of Industrial and Production Engineering, Bangladesh University of Engineering and Technology (BUET), Dhaka -1000, Bangladesh C H R O I C L E A B S T RA C T

Article history:

Received July 22013

Received in revised format

September 72013

Accepted September 272013

Available online

September 272013

Keywords:

Inventory model

Process reliability

Imperfect production process

$E O Q$

$E P Q$

\begin{abstract}
Inventories are the materials stored either waiting for processing or experiencing processing and in some cases for future delivery. Inventories are treated both as blessings and evil. As they are like money placed in a drawer, assets tied up in investments, incurring costs for the care of the stored material and also subject to spoilage and obsolescence there have been a spate of programs developed by industries, all aimed at reducing inventory levels and increasing efficiency on the shop floor. Nevertheless, they do have positive purposes such as stable source of input required for production, less replenishment and may reduce ordering costs because of economies of scale. Finished goods inventories provide for better customer service. So formulating a suitable inventory model is one of the major concerns for an industry. Again considering reliability of any process is an important trend in the current research activities. Inventory models could be both deterministic and probabilistic and both of which must account for the reliability of the associated production process. This paper discusses the major works in the field of inventory modeling driven by reliability considerations, which ranges from the very beginning to latest works just published.
\end{abstract}

\section{Introduction}

Inventory is the stock of any item a company keeps, be it a physical product or a service, to be used in the company's output. Inventories are idle resources tying up a company's capital that could have been put to more profitable use. However, the lack of synchronization in the production system makes holding inventory a necessity. It is thus no surprise to find many managers regard inventories as a necessary evil. Representing a substantial portion of the total assets of a business, inventories are used to serve a variety of the functions chief among which are: (i) coordinating operations. (ii) smoothing production. (ii) achieving economies of scale. and (iv) improving customer service. Two things are very important for any inventory models: when to order and how much to order and the latter is termed as economic order quantity. Traditional approaches to the problem of determining economic ordering

* Corresponding author.

E-mail: imtiazavi@ipe.buet.ac.bd (I. Ahmed)

(C) 2014 Growing Science Ltd. All rights reserved.

doi: $10.5267 /$ j.jijiec.2013.10.001 
quantities for different models of inventory have always assumed implicitly that items produced are of perfect. Product quality, however, is not always perfect, and is usually a function of the reliability of the production process. When the production process is in a good condition, items produced may be of high or perfect quality. As time goes on, the process may deteriorate because of the items produced may contain defectives or items may have substandard quality. The relationship between production lot size and the quality of the product may thus be significant. A larger lot size requires a longer production cycle, and hence is more likely to contain more defective items. This paper addresses the literature review of economic relationships of lot size, quality, set-up and holding costs, demand rate, deterioration (reliability) of the production process. The paper also discusses how different issues affect the overall cost and various methods to explore the optimal result. Specifically, the problem of determining economic production quantities with imperfect production processes is analyzed. A production process may go from an "in-control" state to the "out-of-control" state while producing a lot. At the beginning of the production of the next lot, we assume that the process is restored back to the "in- control" state. This could be due to maintenance measures, which are part of the set-up of the production process. In other words, the setup cost of production includes the cost of restoring the machine to the "good" state. Evidently, the amount of effort spent in this restoration, or set-up stage, may influence the rate of deterioration of the production process while it is producing the lot. The shift in performance of the process is assumed to take place at a random point in time after production has begun. Once the production process is in an "out-of- control" state, it is assumed that a proportion of the items produced are defective. The defective items will eventually be reworked, replaced or passed onto the customers, which could be another production stage using the current output as their input. Of course, in some cases, defective items may not be reworked, and hence will have to be discarded. Under such circumstances, one may look for the production of a larger quantity to meet customers' demand with non-defective products, or result in not meeting some customers' demands. Traditional modeling usually ignores the impact of producing defective items; hence, the reliability of overall process has to be adjusted.

The process reliability depends on a great variety of factors such as production technology, machine capability, jigs and fixtures, work methods, use of on-line monitoring devices, skill level of the operating personnel and inspection, maintenance and replacement policies. Higher reliability means products with acceptable quality are more consistently produced by the process; thereby reducing the costs of scrap and rework of substandard products, wasted materials and labor hours. However, high reliability can be achieved with substantial capital investment, which increases the cost of interest and the depreciation of the production process. In different models such as economic order quantity (EOQ), economic production quantity (EPQ) and stochastic model, researchers include the effects of reliability sometimes with other considerations and sometimes alone and then formulating the cost function based on it and solve the resulted models with different techniques.

The aim of this paper is to review the last 35 years work in the reliability driven inventory modeling field. The paper is organized by dividing more than 30 research papers according to the model used in incorporating reliability. Section 2 presents the approaches to incorporate reliability in EOQ model, section 3 summarizes the major works in reliability driven EPQ model of inventory, section 4 focuses on the effects of reliability in newly developed entropy cost based model and finally section 5 highlighted some other modeling approaches considering reliability. In section 6 , some general insights and future research directions are discussed.

\section{Reliability adjusted EOQ (economic order quantity) model}

Porteus (1986) proposed that lower setup costs could benefit production systems by improving quality control. The EOQ model was considered to validate his proposal. The idea behind this was introducing a simple model that captures a significant relationship between quality and lot size. While producing a lot, the process can go "out of control" with a given probability each time it produces another item. 
Once out of control, the process produces defective units throughout its production of the current lot. The system incurs an extra cost for rework and related operations for each defective piece that it produces. Thus, there is an incentive to produce smaller lots, and have a smaller fraction of defective units. Porteus (1985) therefore first tackled the problem of specifying the optimal lot size that accounts for the consequences of defects. The paper then introduced three options for investing in quality improvements: (i) reducing the probability that the process moves out of control (which yields fewer defects, larger lot sizes, fewer setups, and larger holding costs); (ii) reducing setup costs (which yields smaller lot sizes, lower holding costs, and fewer defects); and (iii) simultaneously using the two previous options. For the first case, only quality percentage is increased without considering setup cost reduction. For a specific form of the investment cost, the optimal solution was explicitly derived. Then opposite was tested. The results of Porteus (1985) were extended for cases of investing when the reducing the setup cost parameter is allowed, without allowing for the simultaneous investment in process quality improvement. The paper assumed the logarithmic form: an investment of $\mathrm{a}_{\mathrm{K}}(\mathrm{K})=\mathrm{A}-$ $\mathrm{B} \ln (\mathrm{K})$ where $\ln (\mathrm{K})$ is required to change the setup cost to $\mathrm{K}$ from the original level of $\mathrm{K}_{0}$. Compared to the basic model, such investment yields a smaller lot size, lower holding cost, and better output quality. Again, by assuming a specific form of the investment cost function, the optimal solution was explicitly obtained. From the above two results it was proved the optimal is somewhere between the two extreme. Finally, the paper combined the options of the previous two approaches and determined the optimal setup cost and process quality level, simultaneously. The results have been broken into four cases, depending on whether or not investment is made for process quality improvement and/or setup cost reduction.

Cheng (1989b) proposed an EOQ model with demand-dependent unit cost and formulate the optimization problem as a geometric program (GP) and finally solved the GP analytically to obtain a closed-form optimal solution. An illustrative example was provided to show the working procedures of applying GP to solve a given problem and reached a decision that GP has potential as a viable mathematical tool for the analysis of a certain class of inventory control problems. Later, Cheng (1991) combined his previous two works (Cheng 1989a, 1989b) for EOQ model. Two major assumptions in the classical EOQ model are that demand is constant and deterministic and that the unit price (unit production cost) is independent of the order (production) quantity. Implicitly, the model also assumes that items produced are of perfect quality. However, in reality, product quality is not always perfect but a function of the reliability of the production process employed to manufacture the products. Consequently, investment in improving the reliability of the production process is the key for achieving consistently a high level of product quality. However, this will increase the fixed cost of production to push up the unit production cost since the costing system, particularly an absorption costing system commonly used in batch manufacturing, will inevitably apportion the increased production overheads to each individual product. In addition, under certain circumstances, the demand rate for a product and its unit production cost are not totally independent. Such a relationship between demand and unit cost exists in two typical situations. First, in a manufacturing environment; when demand is high, a company can produce more items to spread the fixed cost of production more widely, which will result in lower unit production cost. Second, in a distribution environment; if sufficient demand exists, a firm can arrange for longer-term supply contracts with the suppliers to take advantage of a quantity discount on the unit price. Thus, it is quite natural to expect that the unit cost of production is an increasing function of process reliability and a decreasing function of demand rate. In the paper, a model is proposed in which the relationship between unit production cost and process reliability and demand rate are expressed as a general power function. The objective is to minimize the long-term average annual cost, i.e. the sum of setup, unit production and inventory carrying costs over a long period of time, which is a function of process reliability, demand rate, and production quantity and the relevant cost parameters in the present case. The idea of reliability influencing the unit cost function was the same as his previous model for EPQ. However, this time he proposed that the cost function was directly related to demand and the relation function was changed into: $V(D, r)=a^{-b} r^{c}$ for $a, b, c>=0$ where $\mathrm{D}=$ Demand rate; $\mathrm{r}=$ process reliability, finally the cost equation was solved by GP approach. 
Tripathy et al. (2003) extended Cheng's approach by considering demand dependent unit cost and reliability of the production process for the case of EOQ model under a new dimension. He assumed demand exceeds supply and that the unit cost of production was directly related to reliability and inversely to the demand rate. The power equation proposed by him is $\mathrm{P}(\mathrm{r}, \chi)=\mathrm{a}(1-\mathrm{r})^{-\mathrm{b}} \chi^{-\mathrm{c}}$, where $\mathrm{r}=$ production process reliability, $\chi=$ demand rate $\mathrm{P}(\mathrm{r}, \chi)=$ unit production cost and $\mathrm{a}, \mathrm{b}$, c are nonnegative real numbers.

\section{Reliability adjusted EPQ (economic production quantity) model}

Rosenblatt and Lee (1986), two pioneers in reliability-incorporated inventory modeling field, studied the effect of an imperfect production process in a single machine-single product system. The system is assumed to be in an "in-control" state at the beginning of each production cycle, because of some maintenance, which is part of the set-up process. However, the production process may shift to an "outof-control" state during the production run, where the elapsed time till the shift is assumed to be a random variable exponentially distributed with a given mean. The economic production run length for such a system is derived, which is a modification of the classical economic manufacturing quantity (EMQ) formula. Such a production run is found to be shorter than that of the classical formula. It decreases as the defective rate or the cost of defective items increases. The analysis is further extended to incorporate the dynamic nature of deterioration in the production processes. Two different cases are presented: The first case assumed that the process will deteriorate linearly after a certain time. The second case assumed an exponential deterioration. The EMQ formula derived is found to be very robust in both cases, and is thus very useful, especially when the exact parameters for the dynamic deterioration of the process are difficult to estimate. Finally, the model is generalized for a case where there are multiple states of the process, and deterioration follows a step-wise fashion. A necessary and sufficient condition for the economic production run length is derived, which is shown to be smaller than or equal to that of the two-state model. Numerical examples illustrate how the optimal production run length can be derived for the various cases considered

The determination of the most cost-effective production quantity under rather stable conditions is commonly known as the classical economic production quantity (EPQ), with an instantaneous or noninstantaneous receipt inventory problem. Over the last four decades, an abundance of research on this topic has been undertaken and many interesting results have been reported in the literature, see, e.g., Aggarwal (1974), Clark (1972), Hadley and Whitin (1963), Nahmias (1982), Silver (1981), Tinarelli (1983), Veinott (1966), Wagner (1980), Whitin (1954), Taleizadeh et al., (2011) and Hsu (2012). We know that four major assumptions in the classical EPQ model are that (i) demand is constant over time and known with certainty, (ii) no excess stock is carried, and no backorders and lost sales are allowed, (iii) the lead time is zero, and (iv) the unit production cost is independent of the production quantity. Implicitly, the model also assumes that items produced are of perfect quality (Hax \& Canada, 1984). Cheng (1989a) pointed out that classical economic production quantity (EPQ) model's assumption of perfectly reliable production process with a fixed set-up cost is not logical. While the reliability of the production process cannot be perfect without a price, its set-up cost can be reduced with investment in flexibility improvement. Therefore, he proposed a general equation to model the relationship between production set-up cost and process reliability and flexibility. The objective is to minimize the total cost function based on the values of process reliability, flexibility, unit variable production cost, inventory carrying cost and production quantity over a long period of time. In many situations, this is mathematically equivalent to minimizing the total cost per unit time which is generally a simpler performance measure to deal with. In this paper, it is proposed that total cost of interest and depreciation per production cycle is inversely related to set-up cost and directly related to process/production reliability according to the following equation: $\mathrm{Y}(\mathrm{S}, \mathrm{r})=\mathrm{a} \mathrm{S} \mathrm{S}^{-\mathrm{b}} \mathrm{r}^{\mathrm{c}}$ for $\mathrm{a}, \mathrm{b}, \mathrm{c}>=0$ where $S=$ setup cost; $r=$ process reliability, later this equation is taken by many researchers to formulate their model. The total cost function to be minimized is $\mathrm{S}+\mathrm{pq}+\mathrm{Hq}^{2} \mathrm{r}^{2} / 2 \mathrm{D}+\mathrm{Y}(\mathrm{S}, \mathrm{r})$, where $\mathrm{p}=$ unit production cost $\mathrm{q}=$ production quantity per batch $\mathrm{D}=$ demand rate and $\mathrm{H}=$ holding cost per unit per 
time period. Differential calculus is a natural analytical tool for solving this type of optimization problem involving continuous decision variables. However, due to the general nature of the proposed relationship between production set-up cost, process reliability and flexibility, optimization by calculus often leads to a system of non-linear equations, which, in general, are hard to be solved explicitly and numerical methods are always needed to obtain approximate solutions. Consequently, closed-form optimal solutions to this EPQ problem are not easily available using the calculus-based optimization technique. As a result, the inventory optimization problem is formulated as a geometric program (GP) and solved to obtain closed-form optimal solutions. After the theoretical treatment, a numerical example is provided to illustrate how the GP theories are applied to solve a given problem. Finally, some aspects of post-optimality sensitivity analysis based on the GP approach are discussed.

Leung (2007) proposed an EPQ model with reliability and flexibility consideration and solved it with generalized GP. He established more general results using the arithmetic-geometric mean inequality in which a general power function is proposed to model the relationship between production set-up cost (which implicitly measures the degree of process flexibility) and process reliability as independent variables and interest and depreciation cost as a dependent variable. The objective was to minimize the long-run expected average annual cost function, i.e. the sum of set-up, production, inventory holding, and interest and depreciation costs, which is a function of set-up cost, production quantity, process reliability and the relevant cost parameters in the presented case. The assumptions in his paper are similar to those in the study of Gerchak and Parlar (1990) in which it is assumed that quality assurance efforts affect the variance of yield, but differs in Leung (2007) assumed that the efforts affect the mean yield rate (i.e. expected fraction acceptable). Thus, a high level of product quality can only be consistently achieved with substantial investment in improving the reliability of the production process. Furthermore, while the set-up time and hence set-up cost will be fixed in the short term, it will tend to decrease in the long term because of the possibility of investment in new, highly flexible machineries.

Bag et al. (2009) argued that both reliability and flexibility need to be considered during formulation of EPQ model. They stated that though reliability of the production process cannot be increased without a price, its set-up cost can be reduced with investment in flexibility improvement. In the paper, a production inventory model with flexibility and reliability (of production process) consideration is developed in an imprecise and uncertain mixed environment. The aim of this paper is to introduce demand as a fuzzy random variable in an imperfect production process. Here, the set-up cost and the reliability of the production process along with the production period are the decision variables. Due to fuzzy-randomness of the demand, expected average profit of the model is a fuzzy quantity and its graded mean integration value (GMIV) is optimized using unconstraint signomial geometric programming to determine optimal decision for the decision maker (DM). In this work, an EPQ model is considered, where demand of the item is fuzzy random in nature with known probability distribution and the production process is assumed to be not $100 \%$ perfect, i.e. a fraction of the produced items are defective. Further, it is assumed that the defective items are sold at a reduced price and the selling price of fresh units is taken as a mark-up over the unit production cost. The model is formulated to maximize the expected average profit. Panda and Maiti (2009) proposed a multi-item economic production quantity (EPQ) models considering selling price dependent demand, infinite production rate, stock dependent unit production and holding costs. Flexibility and reliability consideration are introduced in the production process. The models are developed under two fuzzy environments; one with fuzzy goal and fuzzy restrictions on storage area and the other with unit cost as fuzzy and possibility /necessity restrictions on storage space. The objective goal and constraint goal are defined by membership functions and the presence of fuzzy parameters in the objective function is dealt with fuzzy possibility/necessity measures. The models are formed as maximization problems. The first one the fuzzy goal programming problem is solved using Fuzzy Additive Goal Programming (FAGP) and modified GP methods. The second model with fuzzy possibility/necessity measures is solved by GP method. The models are illustrated through numerical examples. The sensitivity analyses of the profit function due to different measures o possibility and necessity are performed and presented graphically. 
Krishnamoorthi and Panayappan (2012) pointed out that in the "Economic Production Quantity (EPQ)" model, which is commonly used by practitioners in the fields of production and inventory management to assist them in making decision on production lot size has a common assumption that all units produced are perfect and shortages are not allowed. However, under real world conditions, the defective items will be produced in each cycle of production and shortages and scrap are possible. These assumptions will underestimate the actual required quantity. Hence, the defective items cannot be ignored in the production process. Rework process is necessary to convert those defective into finished goods. Therefore, their study proposed an EPQ model that incorporates both imperfect production quality and falsely not screening out a proportion of defects, thereby passing them onto customers, resulting in defect sales returns. To reach this objective, a suitable mathematical model is developed and the optimal production lot size, which minimizes the total cost.

Mahapatra et al. (2012) pointed out what Cheng (1989b) first found out that a basic assumption in the classical EPQ model is that the production setup cost is fixed. In addition, the model also implicitly assumes that items produced are of perfect quality. However, in reality product quality is not always perfect but directly affected by the reliability of the production process employed to manufacturer the product. Thus, a high-level of product quality can only be consistently achieved with substantial investment in improving the reliability of production process. They developed an EPQ model with demand dependent unit production cost in fuzzy environment. Flexibility and reliability consideration are introduced in the production process. The models are developed under fuzzy goal and fuzzy restrictions on budgetary cost. The inventory related costs and other parameters are taken as fuzzy in nature. The problem is solved by parametric GP technique. The result obtained by fuzzy EPQ model is compared with the nonlinear programming. Shah and Soni (2011) developed an EPQ model with flexibility and reliability consideration of production process in an imprecise and uncertain mixed environment. The model incorporated fuzzy random demand, an imprecise production preparation time and shortage. Here, the setup cost and the reliability of the production process along with the backorder replenishment time and production run period are the decision variables. Due to fuzzy-randomness of the demand, expected average demand is a fuzzy quantity and imprecise preparation time is represented by fuzzy number. Therefore, both are first transformed into a corresponding interval number and then using the interval arithmetic, the single objective function for expected profit over the time cycle is changed to respective multi-objective functions. Due to highly nonlinearity of the expected profit functions it is optimized using a multi-objective genetic algorithm (MOGA). The associated profit maximization problem is illustrated by numerical examples and its sensitivity analysis is carried out.

\section{Reliability adjusted "Entropy cost based" model}

Jaber et al. (2004) postulated that a production system resembles a physical system operating within surroundings, which include the market and the supply system. Physical thermodynamic system is defined by its temperature, volume, pressure and chemical composition. A system is in equilibrium when these variables have the same values at all points. A production system could be described analogously by its characteristics, for example the price $(\mathrm{P})$ that the system ascribes to the commodity (or collection of commodities) that it produces. Reducing the price of the commodity below the market price may increase demand and produce a commodity flow (sales) from the system to its surroundings. This is similar to the flow of heat from a high temperature (source) to a low temperature (sink) in a thermodynamic stem, where part of this heat is converted into useful work and some of the heat is lost from the system and wasted. Analogously, some of the commodity is sold to the market and is converted to revenue (equivalent to useful work) and some is wasted. They suggested that production system performance could be improved by applying the first and second laws of thermodynamics to reduce system entropy. They suggested using the concept of entropy cost to account for the hidden costs inherent in management systems.

Later Jaber et al. (2009) combined their entropy cost concept with imperfect production process. However, unlike the commodity flow strategies assumed in the earlier papers, this paper has presented a commodity flow policy that is more consistent with the EOQ models. The results suggested that 
ignoring entropy cost may result in more expensive commodity control policies and it is cheaper to control larger batch sizes than is to control a larger number of smaller batches for both perfect and imperfect processes. The results indicated that it is more relevant to account for entropy cost for expensive rather than for inexpensive products. The results also indicated that it is more expensive to control the flow of a single unit of commodity for an imperfect process, i.e. a process generating defects requiring rework (Porteus, 1986), than is for a perfect process as assumed in the classic EOQ model. The results further suggest/indicated that a firm, which is unable to estimate its cost parameters properly, may find that ordering in larger lots is an appropriate policy to counter entropy effects.

Ameli et al. (2013) extended the EnOQ model proposed by Jaber et al. (2004) by considering deteriorating items with imperfect quality and price dependent demand. They also assumed fuzzy inflation and discount rates. Imperfect items with deterioration were shown in industries such as electronic, agriculture and food industries. On the other hand, the major loss due to inflation is caused by its uncertainty instead of its high rate. Uncertainty in future inflation rate rises the inflation rate and in turn high inflation rate increases uncertainty in future inflation rate. So due to the world's current uncertain conditions it is important to consider uncertainty in inflation rate to capture the real world better. Hence, in this paper, a new thermodynamics approach model under fuzzy inflationary conditions, fuzzy time discounting for defective items with constant defective and deterioration rate and time dependent demand has been proposed. A mathematical model is developed to determine the number of cycles that maximizes the present value of total revenue in a finite planning horizon. The fuzzified model for inflation and discount rate is solved by two methods: signed distance and fuzzy numbers ranking. Numerical examples are presented and results are discussed. Results showed that the number of cycles decreases in fuzzy inflationary conditions. They also illustrated that defuzzification method results in more cycles than fuzzy method.

\section{Other modeling approaches incorporating reliability}

Prekopa (1965) introduced reliability in inventory model and provided its asymptotic solution and later Prekopa and Kelle (1978) proposed three reliability type inventory models based on stochastic programming which are basically generalization of his previous models and also provided their solution. He used nonlinear programming for solution with one probabilistic constraint. Simulation is used to determine the value of constraining function. Model 1 has some assumptions about delivery process like number of delivery times; total amount delivered is constant, existence of a smallest amount to be delivered. Another one is random vector of the components of which are the random delivered amount is stochastically independent of the random vector of the delivery time points. The

$2^{\text {nd }}$ model differs from the first one that it has an added constraint considering conditional expectation appear. It considers not only the rarity of the occurrence of the unsatisfied demand but also upper bound for the average magnitude of the unsatisfied demand. It has an assumption that no demand is lost. Moreover the time interval in which unsatisfied demand exists is proportional to the magnitude of violation. Model 3 consists of a penalty term and contains the previous models as special cases. It is considered as one of the earliest work in the reliability driven inventory model field.

Louit and Pascual (2011) presented various approaches for the determination of the optimal stock size, when the stock is composed of (i) non-repairable or (ii) repairable parts. The paper is focused on spares for relatively expensive, highly reliable components, rather than on fast-moving spare parts. Optimization criteria considered are minimization of costs, maximization of equipment availability, and the achievement of a desired stock reliability (probability that a spare part request will not be rejected due to lack of spares in stock). For stock reliability, instantaneous and interval reliability calculations are considered. In addition, models directed to the estimation of the remaining life of a given stock of spare parts (at a certain stock reliability level) are introduced. The paper presented several models for spare parts inventory optimization from a "reliability engineering perspective" and illustrated their use through industrial case studies. This perspective differs from that of general inventory control primarily 
in the sense that no infinite populations are assumed, thus the demand rate for spare depends on the number of units currently in operation. Two kinds of reliability are introduced here one is instantaneous reliability this is the probability that a spare is available at any given moment in time. It is equivalent to the fraction of demands that can be immediately satisfied from stock at hand. In the literature, instantaneous reliability is commonly referred to as fill rate or less often as availability of stock or point availability in the long run. Another is Interval reliability this is the probability of not running out of stock at any moment over a specified period of time, such as one year. Because reliability has to be maintained for every moment during the interval, this criterion is more demanding than instantaneous reliability. In case of repairable components both limited and unlimited repair capacity is considered. The paper described all of these models, which are subject to practical industrial application and presented case studies from utilities and mining to illustrate their use.

Krishnamoorthi (2011) investigated the effect of quality cost on inventory control policies in a manufacturing system during the product life cycle, which consists of introduction, growth and decline stages. The defective rate is considered as a variable of known proportions. An inventory model in order to minimize the total cost of inventory and integrated with cost of quality is developed. The objective is to minimize the overall total relevant inventory cost. An exact mathematical model and a solution procedure are established. Necessary and sufficient conditions for a unique and global optimal solution are derived. An illustrative example is provided and numerically verified. This seems to be the first time where such an inventory model for product life cycle is mathematically treated and numerically verified. It is concluded that there is (i) direct relationship between rate of defective items with optimal quantity, cycle time and total cost and also an inverse relationship between rate of defective items and order cost, holding cost and demand down time, (ii) direction relationship between rate of quality cost with optimum quantity, cycle time, holding cost and total cost but inverse relationship with ordering cost.

Tripathy and Pattnaik (2011) pointed out that as markets become more and more competitive, reliability has become a prevailing characteristic of the modern production systems operating in complex, dynamic and uncertain environments. Ensuring reliability for exceptional products requires stringent production control measures often with costs that are usually difficult to estimate. Yet not accounting for these in costs can lead to less efficient production systems. Therefore, they suggested that the need to consider reliability and need for instantaneous receipt resembles an imperfect production process. In the paper, the applicability of these factors is demonstrated in a simple reverse inventory model with an imperfect production process, where the unit cost of production is inversely related to reliability and directly related to demand. These objective functions are included to model the problem accurately. Under reasonable conditions, maximum positive cost savings are achieved when process reliability increases. They identified their intention to find an optimal inventory policy with reliability considerations where demand was deterministic and production unit cost depends on process reliability and demand rate. The company usually has a specified level of reliability for the production process, which depends on a number of different factors. However, reliability is achieved with additional fixed and variable costs, which pushes up the production unit cost. Using the proposed model, if the production unit cost is inversely associated with reliability and directly associated with demand, a significant amount of savings can be achieved

Mirzahosseinian and Piplani (2011) model the PBL system as a queuing network by enhancing the classical repairable parts inventory model. They model the closed-loop inventory system as an $\mathrm{M} / \mathrm{M} / \mathrm{m}$ queue in which component failures are Poisson distributed and the repair times at the service facility are exponential. They justified their model improves upon the classical model by relaxing some restrictive assumptions, such as fixed failure rate, fixed repair rate and infinite capacity at repair facility. The model focused on the interaction between inventory management, component reliability and repair facility efficiency. The model was then analyzed to determine the factors that have significant impact on the system availability. The results showed that the base stock level of the spare parts has negligible 
effect on the system availability. Thus, to achieve a desired availability level, the supplier has to improve the component reliability and the repair time, rather than invest in building up a stock of spares. In addition, they formulated two metrics that allow the customer to monitor the key parameters affecting system availability. Furthermore, the model recommended concentrating on the component reliability and repair system efficiency to improve the availability of the system with repairable spare parts. They identified optimizing the cost for a repairable inventory system in order to find the optimal failure rate, server repair rate and the number of servers is a potential area for future research. Modeling such a system would be easier if the base stock were fixed at a constant value, as it does not seem to have an effect on system availability.

\section{Conclusion}

In reality, there is nothing like perfect production system and it is important to incorporate reliability in any inventory modeling regardless of its type. This paper has summarized the research works accomplished in inventory modeling for an imperfect production process for the last 35 years. These research outcomes have been analyzed and separated based on the basic modeling approach in which the reliability adjustments are made. The basic models used in these works are mainly EOQ, EPQ, entropy cost based model, stochastic model and some other approaches like spare parts inventory model, model specific to product life cycle stage and some more. In formulation of the model, reliability is adjusted with different approaches and forms of equation. The optimum cost has been determined by solving the cost model subjected to some constraints with numerous approaches like geometric programming, differential calculus, multi-objective genetic algorithm, fuzzy additive goal programming, modified geometric programming, etc. One interesting aspect is that most of the works reviewed here considered traditional EOQ and EPQ model for reliability adjustment and there are few approaches, which are out of the box and considered probabilistic and advanced inventory model for adjustment. So in future, researchers could extend their works to model like Wagner-Whitin algorithm, newsvendor model, base stock model, $(\mathrm{Q}, \mathrm{R})$ model, etc.

\section{References}

Ameli, M., Mirzazadeh, A., \& Shirazi, M.A. (2013). Entropic Economic Order Quantity model for items with imperfect quality considering constant rate of deterioration under fuzzy inflationary conditions. International Journal of Industrial Engineering \& Production Research, 24(1), 91-99

Aggarwal, S.C. (1974). A review of current inventory theory and its applications. International Journal of Production Research, 2, 443-482.

Bag, S., Chakraborty, D., \& Roy, A.R. (2009). A production inventory model with fuzzy random demand and with flexibility and reliability considerations. Computers and Industrial Engineering, $56,411-416$

Cheng, T. (1989a). An economic production quantity model with flexibility and reliability consideration. European Journal of Operational Research, 39, 174-179.

Cheng, T. (1991). EPQ with process reliability and quality assurance considerations. Journal of the Operational Research Society, 42, 713-720.

Cheng, T. (1989b). An economic order quantity model with demand dependent unit cost. European Journal of Operational Research, 40, 252-256.

Clark, A.J. (1972). An informal survey of multi-echelon inventory theory. Naval Research Logistics Quarterly, 19, 621-650.

Gerchak, Y., \& Parlar, M. (1990). Yield randomness, cost tradeoffs and diversification in the EOQ model. Naval Research Logistics, 37, 341-354.

Hax, A.C., \& Canada, D. (1984). Production and Inventory Management. Prentice-Hall, New Jersey.

Hadley, G., \& Whitin, T.M. (1963). Analysis of Inventory Systems. Prentice-Hall, New Jersey.

Hsu, L. (2012). A note on "An economic order quantity (EOQ) for items with imperfect quality and inspection errors". International Journal of Industrial Engineering Computations, 3(4), 695-702. 
Jaber, M., Bonney, M., \& Moualek, I. (2009). An economic order quantity model for an imperfect production process with entropy cost. International Journal of Production Economics, 118, 26-33.

Jaber, M.Y., Nuwayhid, R.Y., Rosen, M.A., (2004). Price-driven economic order systems from a thermodynamic point of view. International Journal of Production Research, 42:5167-5184.

Krishnamoorthi, C., \& Panayappan, S. (2012). An EPQ model with imperfect production systems with rework of regular production and sales return. American Journal of Operations Research, 2, 225234.

Krishnamoorthi, C. (2011). The effect of quality cost on inventory model for product life cycle with growth stage and defective items. International Journal of Pure and Applied Mathematics, 71(1), 117

Louit, D., Pascual, R., Banjevic, D., \& Jardine, K.S. (2011). Optimization models for critical spare parts inventories-A reliability Approach. Journal of the Operational Research Society, 62(6), 992-1004

Leung K-N.F. (2007). A generalized geometric programming solution to an economic production quantity model with flexibility and reliability considerations. European Journal of Operational Research, 176, 240-251.

Mahapatra, G.S., Mandal, T.K., \& Samanta, G.P. (2012). Fuzzy parametric geometric programming with application in fuzzy EPQ model under flexibility and reliability consideration. Journal of Information and Computing Science, 7(3), 223-234

Mirzahosseinian, M., \& Piplani, R. (2011). A study of repairable parts inventory system operating under performance-based contract. European Journal of Operational Research, 214, 256-261.

Nahmias, S. (1982). Perishable inventory theory: A review. Operations Research, 30, 680-708.

Porteus, E.L. (1985). Investing in reduced set-ups in the EOQ model. Management Science, 31, 9981010.

Porteus, E. (1986). Optimal lot sizing, process quality improvement and setup cost reduction. Operations Research, 34, 137-144.

Prekopa, A., \& Kelly, P. (1978). Reliability Type inventory modeling based on stochastic programming. Mathematical Programming Study, 9, 43-58.

Prekopa, A. (1965). Reliability equation for an inventory problem and its asymptotic solutions. Colloquium on Application of Mathematics to Economics, 1, 317-327.

Panda, D., \& Maiti M. (2009). Multi-item inventory models with price dependent demand under flexibility and reliability consideration and imprecise space constraint: A geometric programming approach. Mathematical and Computer Modeling, 49, 1733-1749.

Rosenblatt, M., \& Lee, H. (1986). Economic production cycles with imperfect production processes. International Industrial Engineers Transactions, 18(1), 48-55.

Silver, E.A. (1981). Operations research in inventory management: A review and critique. Operations Research, 29(4), 628-645.

Taleizadeh, A., Widyadanab, G., Wee, H., \& Biabanid, J. (2011). Multi products single machine economic production quantity model with multiple batch size. International Journal of Industrial Engineering Computations, 2(2), 213-224.

Tripathy, P., Wee, W., \& Majhi, P. (2003). An EOQ model with process reliability consideration. Journal of Operational Research Society, 54, 549-554.

Tinarelli, G.U. (1983). Inventory control: Models and problems. European Journal of Operational Research, 14, 1-12.

Tripathy, P.K., \& Pattnaik, M. (2011). Optimal inventory policy with reliability consideration and instantaneous receipt under imperfect production process. International Journal of Management Sciences and Engineering Management, 6(6), 413-420

Wagner, H.M. (1980). Research portfolio for inventory management and production planning systems. Operations Research, 28(3), 445-475.

Whitin, T.M. (1954). Inventory control research: A survey. Management Science, 1(1), 32-40.

Veinott, A.F. (1966). The status of mathematical inventory theory. Management Science, 12(11), 745777. 